\title{
Javanese Lexical Variation of Cirebon Dialect in Pabuaran and Ciperna Region
}

\author{
Eva Utami Durahman ${ }^{1}$, Dinny Sofiyani ${ }^{2}$, Yosi Ayu Pratiwi ${ }^{3}$, Fani Setiani ${ }^{4}$ \\ utamieva08@gmail.com ${ }^{1}$, dinysofiyani@gmail.com ${ }^{2}$, yosiayupratiwi@gmail.com ${ }^{3}$, \\ fanisetiani2407@gmail.com ${ }^{4}$ \\ STIBA Invada Cirebon ${ }^{1,2,4}$ \\ STKIP Invada Cirebon ${ }^{3}$
}

\begin{abstract}
This study highlights the lexical differences phenomena in Pabuaran and Ciperna region which is located in Cirebon Regency of West Java. This study aims: 1) to describe the Javanese lexical variations of Cirebon dialect in Pabuaran and Ciperna region, 2) and to find out whether those variations are dialect, subdialect, or only speech level differences of Cirebon dialect itself by utilizing dialectometric method. The result shows that there are 108 lexical differences in the form of pronouns (10), nouns (26), verbs (44), adjectives (14), and adverb (14) in Pabuaran and Ciperna region. This result is obtained by using 207 words Swadesh List and 144 additional words submitted as questions to the local people. Furthermore, after being calculated by dialectometric method, it can be concluded that those lexical variation in Pabuaran and Ciperna are chategorized as subdialect rather than dialect or speech level differences.
\end{abstract}

Keywords: Cirebon dialect, Javanese language, lexical variations

\section{Introduction}

Indonesia has 560 local languages along with their own dialects, one of them is Javanese language. It is one of Indonesian local language used by most of Indonesian people. Javanese language is the $11^{\text {th }}$ language which has the most active speakers in the world that reach 84,3 million (Purwaningsih, 2017). Javanese language develops in many Indonesian regions, its development causes the language variation which is also called dialect, such as Javanese language dialect in Cirebon.

Cirebon Regency is located in the eastern part of West Java. Geographically, Cirebon regency is bordered by another regency. Most people of those regions are the native speaker of Javanese and Sundanese language. The impact of this geographical position causes language variation or dialect in Cirebon. In this study, the authors choose two region from the two different sub-district in Cirebon regency as the research center, those are Pabuaran and Ciperna.

Table 1.1 Cirebon Regency border area and its majority language

\begin{tabular}{|l|l|l|}
\hline North & Indramayu Regency & Javanese Language \\
\hline South & Kuningan Regency & Sundanese Language \\
\hline East & $\begin{array}{l}\text { Cirebon City, and Brebes Regency (Central } \\
\text { Java) }\end{array}$ & $\begin{array}{l}\text { Javanese and } \\
\text { Sundanese language }\end{array}$ \\
\hline West & Majalengka Regency & Sundanese Language \\
\hline
\end{tabular}

Ciperna is located in Talun sub-district in the southern part of Cirebon, close to Penggung region where most people speak Javanese language. It is also close to Beber region nearby Kuningan Region where most people speak Sundanese language. Pabuaran is located 
in the eastern part of Cirebon Regency. As in Ciperna, there is a language contact between Javanese and Sundanese language here. This condition motivates the authors to study how geographical position affects Javanese lexical variation in Pabuaran and Ciperna.

Based on the phenomena described above, the aims of this study are: 1) describing the Javanese lexical variations of Cirebon dialect in Pabuaran and Ciperna region, 2) finding out whether those variations are dialect, subdialect, or only speech level differences of Cirebon dialect itself by utilizing dialectometric method.

This study is conducted under the motivation by several previous studies that had been done by other researchers about lexical variation. The two of them are (1) Variasi Leksikal Bahasa Minangkabau di Kabupaten Pesisir Selatan conducted by Meksi Rahma Nesti (2016) that found 271 lexical variation from 531 questions submitted to the informant in Pesisir Selatan region of Minangkabau and (2) Variasi Leksikal Bahasa Bali Dialek Kuta Selatan conducted by Putu Devu Maharani and Komang Dian Puspita Candra (2018) who discovered several lexical variations in pronoun, noun, verb, adjective, and adverb in Balinese language. The study shows that noun is the most dominant word class found in Balinese language variation.

\section{Theory and Method}

Chaer (2010:62-63) stated that dialect is a language variation of certain speakers that different from one area to another. Dialect can be distinguished based on its lexical, semantic, and phonological aspects. Wardaugh (2002:43-49) classifies dialect into two types; regional dialect and social dialect. Regional dialect is a dialect based on geographical location while social dialect is a dialect related to some factors such as social class, religion, or ethnicity. However, this study limited only to lexical variation of Javanese dialect in Cirebon especially in Pabuaran and Ciperna region.

This study uses descriptive qualitative approach that solve the research problem by describing the selected object based on the facts or data which exist at the exact moment (Nawawi cited in Rina \& Mariati, 2018). The data are collected from the selected informants, in this case as the native speaker of Javanese dialect in Pabuaran and Ciperna, Cirebon. The instrument used to collect the data are 207 basic words in Swadesh List and 144 additional words. The collected data are chategorized based on their word class to find out the lexical variation in both regions and calculated by dialectometric formula to obtain the percentage to determine whether it is a dialect, subdialect, or speech level differences. The percentage of dialectometric formula according to Mahsun (cited in Biantoro, 2009), are as follow:
a. $65 \% \geq$
: assumed as language differences
b. $45-64 \%$
: assumed as dialect differences
c. $25-44 \%$
: assumed as sub dialect differences
d. $10-24 \%$
: assumed as speak differences
e. $9 \% \leq$
: assumed as no differences.

\section{Findings and Discussion}

The study of Javanese dialect in Pabuaran and Ciperna by using 207 basic vocabulary list by Morish Swadesh and 144 additional words found 243 same words and 108 different words used in Pabuaran and Ciperna region as explain in the following sub. 


\section{Lexical Variation}

The study of Javanese dialect in Pabuaran and Ciperna by using 207 basic vocabulary list by Morish Swadesh and 144 additional words found 243 same words and 108 different words used in Pabuaran and Ciperna region as explain in the following sub.

\section{Pronoun}

The study found 10 same words and 10 different words in the form of pronouns (including address terms) used in Pabuaran and Ciperna. Those words are displayed in the table below:

Table 3.1 Pronoun

\begin{tabular}{|c|l|l|l|}
\hline No. & \multicolumn{1}{|c|}{ MEANING } & \multicolumn{1}{|c|}{$\begin{array}{c}\text { PABUARAN } \\
\text { DIALECT }\end{array}$} & \multicolumn{1}{c|}{ DIPERNACT } \\
\hline 1. & aku & nyong & isun \\
\hline 2. & kamu & koen & ira \\
\hline 3. & itu & kue & ikuh \\
\hline 4. & nenek & mide & ema \\
\hline 5. & ipar & ipar & ipe \\
\hline 6. & ibu & mimih & mimi \\
\hline 7. & dia & kaé & dewéké \\
\hline 8. & kami, kita & kita & kita-kitaan \\
\hline 9. & kalian & kabéhané & ira kabéh \\
\hline 10. & ini & kie & kih \\
\hline 11. & mereka & - & deweke kabeh \\
\hline 12. & disini & ningkene & ningkene \\
\hline 13. & disana, disitu & ningkana, ningkono & ningkana \\
\hline 14. & tante & bibi & bibi \\
\hline 15. & kakek & abah & abah \\
\hline 16. & pakde & uwa & uwa \\
\hline 17. & kakak & kakang & kakang \\
\hline 18. & adik & adi & adi \\
\hline 19. & paman & mamang & mamang \\
\hline 20. & ayah & mama & mama \\
\hline & & & \\
\hline
\end{tabular}

\section{Noun}

The study found 98 same words and 26 different words in the form of nouns used in Pabuaran and Ciperna. Those words are displayed in the table below:

Table 3.2 Noun

\begin{tabular}{|l|l|l|l|}
\hline No. & \multicolumn{1}{|c|}{ MEANING } & \multicolumn{1}{|c|}{$\begin{array}{c}\text { PABUARAN } \\
\text { DIALECT }\end{array}$} & \multicolumn{1}{c|}{$\begin{array}{c}\text { DIPERNALECT } \\
\text { DIAĹl| }\end{array}$} \\
\hline 1. & lantai & téhel & jogan \\
\hline 2. & pohon & wit & witwitan \\
\hline 3. & pakaian & klambi & pakean \\
\hline 4. & hutan & hutan & alas \\
\hline 5. & kain lap & lap & serbet \\
\hline 6. & daerah & daerah & jagat \\
\hline 7. & topi & topi & topong \\
\hline 8. & gayung & batok & cibuk \\
\hline 9. & untuk & kanggo & nggo \\
\hline 10. & kambing & embe & wedus \\
\hline 11. & teriak & gowokan & gerowokan \\
\hline 12. & lipstick & abang lambe & benges \\
\hline 13. & akhir & ahir & akhir \\
\hline
\end{tabular}




\begin{tabular}{|c|c|c|c|}
\hline 14. & keset & seset & kesed \\
\hline 15. & anjing & asu & anjing \\
\hline 16. & akar & oyot & oyod \\
\hline 17. & lemak & gaji & lemak \\
\hline 18. & tanduk & sungu & tanduk \\
\hline 19. & bulu & wulu & bulu \\
\hline 20. & sayap & séwiwi & elar \\
\hline 21. & punggung & boyok & gigir \\
\hline 22. & sungai & wangan & kali \\
\hline 23. & danau & waduk & tlaga \\
\hline 24. & debu & awu & kébul \\
\hline 25. & asap & kébul & asép \\
\hline 26. & jalan & dalan & jalan \\
\hline 27. & awal & awal & awal \\
\hline 28. & jendela & gendela & gendela \\
\hline 29. & sepeda & pit & pit \\
\hline 30. & pagar & rajég & rajég \\
\hline 31. & kipas angin & ilir & ilir \\
\hline 32. & sendawa & atob & atob \\
\hline 33. & jari & jeriji & jeriji \\
\hline 34. & tanaman & tanduran & tanduran \\
\hline 35. & duri & eri & eri \\
\hline 36. & selimut & kemul & kemul \\
\hline 37. & pisau & lading & lading \\
\hline 38. & cabe & sabrang & sabrang \\
\hline 39. & kerudung & kudung & kudung \\
\hline 40. & ulat & uler & uler \\
\hline 41. & bibir & lambe & lambe \\
\hline 42. & pisang & gedang & gedang \\
\hline 43. & genting & gendeng & gendeng \\
\hline 44. & bedak & pupur & pupur \\
\hline 45. & pintu & lawang & lawang \\
\hline 46. & jalan raya & dalan & dalan \\
\hline 47. & lingkaran & bunderan & bunderan \\
\hline 48. & katak & kodok & kodok \\
\hline 49. & hantu & setan & setan \\
\hline 50. & layang-layang & layangan & layangan \\
\hline 51. & tikus & curut & curut \\
\hline 52. & zuhur & bedug & bedug \\
\hline 53. & lebaran & rayaan & rayaan \\
\hline 54. & depan & arep & arep \\
\hline 55. & belakang & buri & buri \\
\hline 56. & keriput & kisut & kisut \\
\hline 57. & teman & batur & batur \\
\hline 58. & perempuan, wanita & wadon & wadon \\
\hline 59. & laki-laki, pria & lanang & lanang \\
\hline 60. & manusia & menusa & menusa \\
\hline 61. & anak & bocah & bocah \\
\hline 62. & istri & rabi & rabi \\
\hline 63. & suami & laki & laki \\
\hline 64. & binatang & satoan & satoan \\
\hline 65. & ikan & iwak & iwak \\
\hline 66. & burung & manuk & manuk \\
\hline 67. & kutu & tuma & tuma \\
\hline 68. & ular & ula & ula \\
\hline 69. & cacing & cacing & cacing \\
\hline 70. & ranting & - & batang \\
\hline
\end{tabular}




\begin{tabular}{|c|c|c|c|}
\hline & buah & buah & buah \\
\hline 72. & biji & wijil & wijil \\
\hline 73. & daun & godong & godong \\
\hline 74. & pepagan & - & batang wiwitan \\
\hline 75. & bunga & kembang & kembang \\
\hline 76. & rumput & suket & suket \\
\hline 77. & tali & tali & tali \\
\hline 78. & kulit & kulit & kulit \\
\hline 79. & daging & daging & daging \\
\hline 80. & darah & gétih & gétih \\
\hline 81. & tulang & balung & balung \\
\hline 82. & telur & endog & endog \\
\hline 83. & ekor & buntut & buntut \\
\hline 84. & rambut & rambut & rambut \\
\hline 85. & telinga & telinga & telinga \\
\hline 86. & kepala & endas & endas \\
\hline 87. & mata & mata & mata \\
\hline 88. & hidung & cungur & cungur \\
\hline 89. & mulut & cangkém & cangkém \\
\hline 90. & gigi & untu & untu \\
\hline 91. & lidah & ilat & ilat \\
\hline 92. & kuku & kuku & kuku \\
\hline 93. & kaki & sikil & sikil \\
\hline 94. & tungkai & |- & tungkai \\
\hline 95. & lutut & déngkul & déngkul \\
\hline 96. & tangan & tangan & tangan \\
\hline 97. & perut & wéténg & wéténg \\
\hline 98. & usus & usus & usus \\
\hline 99. & leher & gulu & gulu \\
\hline 100 & payudara & susu & susu \\
\hline 101 & jantung & jantung & jantung \\
\hline 102 & hati & ati & ati \\
\hline 103 & hari & dina & dina \\
\hline 104 & tahun & taun & taun \\
\hline 105 & nama & aran & aran \\
\hline 106 & matahari & matahari & matahari \\
\hline 107 & bulan & wulan & wulan \\
\hline 108 & bintang & lintang & lintang \\
\hline 109 & air & banyu & banyu \\
\hline 110 & hujan & udan & udan \\
\hline 111 & laut & laut & laut \\
\hline 112 & garam & uyah & uyah \\
\hline 113 & batu & watu & watu \\
\hline 114 & pasir & lemah & lemah \\
\hline 115 & bumi & bumi & bumi \\
\hline 116 & awan & awan & awan \\
\hline 117 & kabut & kabut & kabut \\
\hline 118 & langit & langit & langit \\
\hline 119 & angin & angin & angin \\
\hline 120 & salju & salju & salju \\
\hline 121 & & es & es \\
\hline 122 & api & géni & géni \\
\hline 123 & abu & abu & abu \\
\hline 124 & gunung & gunung & gunung \\
\hline
\end{tabular}




\section{Adverb}

The study found 24 same words and 14 different words in the form of adverbs used in Pabuaran and Ciperna. Those words are displayed in the table below:

Table 3.3 Adverb

\begin{tabular}{|c|c|c|c|}
\hline No. & MEANING & $\begin{array}{c}\text { PABUARAN } \\
\text { DIALECT }\end{array}$ & CIPERNA DIALECT \\
\hline 1. & mungkin & ndean & kayae \\
\hline 2. & masa & ilok & masa \\
\hline 3. & tidak ada & langka & laka \\
\hline 4. & baru saja & tembéké & tembé \\
\hline 5. & ya sudah & ya wis & ya uis \\
\hline 6. & besok & ngko esuk & sakiki \\
\hline 7. & ayo & gian & gagian \\
\hline 8. & dimana & ning endi & ning ndi \\
\hline 9. & mengapa & kenang apa & napa \\
\hline 10. & sebelum & sedurung & sedurunge \\
\hline 11. & sesudah & sa uwis & seuwise \\
\hline 12. & beberapa & semene & pira \\
\hline 13. & dengan & bari & karo \\
\hline 14. & dan & karo & lan \\
\hline 15. & kemarin & wingi & wingi \\
\hline 16. & kurang & kurang & kurang \\
\hline 17. & sekarang & kien & kien \\
\hline 18. & lusa & emben & emben \\
\hline 19. & malam & bengi & bengi \\
\hline 20. & siang & awan & awan \\
\hline 21. & kesana & mana & mana \\
\hline 22. & kesini & mene & mene \\
\hline 23. & berapa & pira & pira \\
\hline 24. & sebentar & sedelat & sedelat \\
\hline 25. & pernah & pernah & pernah \\
\hline 26. & kenyang & wareg & wareg \\
\hline 27. & jarang & jarang & jarang \\
\hline 28. & siapa & sapa & sapa \\
\hline 29. & apa & apa & apa \\
\hline 30. & kapan & kapan & kapan \\
\hline 31. & bagaimana & priben & priben \\
\hline 32. & bukan, tidak & dudu, belih & dudu \\
\hline 33. & semua & kabeh & kabeh \\
\hline 34. & banyak & akeh & akeh \\
\hline 35. & $\mathrm{di}$ & ning & ning \\
\hline 36. & dalam & jéro & jéro \\
\hline 37. & kalau & lamun & lamun \\
\hline 38. & karena & soalé & soalé \\
\hline
\end{tabular}

\section{Verb}

The study found 42 same words and 44 different words in the form of verbs used in Pabuaran and Ciperna. Those words are displayed in the table below:

Table 3.4 Verb

\begin{tabular}{|c|l|l|l|}
\hline No. & \multicolumn{1}{|c|}{ MEANING } & \multicolumn{1}{|c|}{$\begin{array}{c}\text { PABUARAN } \\
\text { DIALECT }\end{array}$} & \multicolumn{1}{c|}{$\begin{array}{c}\text { CIPERNA } \\
\text { DIALECT }\end{array}$} \\
\hline 1. & duduk & jagong & ndodok \\
\hline 2. & kerja & mekaya & menggawe \\
\hline
\end{tabular}




\begin{tabular}{|c|c|c|c|}
\hline 3. & cari & luruh & luru \\
\hline 4. & ketawa & gemuyu & ngekek \\
\hline 5. & kencing & nyuyuh & nyuyu \\
\hline 6. & mohon & gona rong gona & nyuwun \\
\hline 7. & pinjam & nyilih & nyili \\
\hline 8. & terpeleset & kesledat & kepeleset \\
\hline 9. & tenggelam & kelelep & kelem \\
\hline 10. & loncat & anjlog & mencelat \\
\hline 11. & menyimpan & dalah & naro \\
\hline 12. & mengikuti & ngintil & ngintili \\
\hline 13. & hisap (menghisap) & nyedot & jilat \\
\hline 14. & ludah (meludah) & idu (nyidu) & ngedoni \\
\hline 15. & muntah & mutah & muta \\
\hline 16. & tiup (meniup) & damu & képus \\
\hline 17. & dengar (mendengar) & rungu & ngerongoknang \\
\hline 18. & bunuh (membunuh) & pateni & dipateni \\
\hline 19. & kelahi (berkelahi) & gulét & tukaran \\
\hline 20. & buru (memburu) & newak & buru \\
\hline 21. & pukul (memukul) & gépuk & gébuk \\
\hline 22. & belah (memotong) & sigar & belah \\
\hline 23. & tusuk (menusuk) & nujlep & tusuk \\
\hline 24. & garuk (menggaruk) & kukur & garuk \\
\hline 25. & gali (menggali) & ngeduk & kéduk, dikéduk \\
\hline 26. & renang (berenang) & ngoyok & adus-adusan \\
\hline 27. & baring (berbaring & gleléngan & turuan \\
\hline 28. & belok (berbelok) & belok & mengkol \\
\hline 29. & beri (memberi) & nei & pai \\
\hline 30. & pegang (memegang) & nyekél & ngemék \\
\hline 31. & peras (memeras) & merés & perés \\
\hline 32. & gosok (menggosok) & gusrék & gosok \\
\hline 33. & cuci (mencuci) & ngumbah & basuh \\
\hline 34. & hapus (menghapus) & pupus & apus \\
\hline 35. & lempar (melempar) & elung & balang \\
\hline 36. & ikat (mengikat) & naléni & taléni \\
\hline 37. & jahit (menjahit) & njait & jait \\
\hline 38. & hitung (menghitung) & ngitung & itung \\
\hline 39. & kata (berkata) & jarehe & ngucap \\
\hline 40. & apung (mengapung) & ngambang & ngapung \\
\hline 41. & air (mengalir) & ngegrojog & banyu (ngalir) \\
\hline 42. & beku (membeku) & beku & atos \\
\hline 43. & bengkak (membengkak) & abuh & abu \\
\hline 44. & bakar & bakar & obong \\
\hline 45. & pergi & lunga & lunga \\
\hline 46. & bangun & tangi & tangi \\
\hline 47. & ambil & jukut & jukut \\
\hline 48. & mandi & adus & adus \\
\hline 49. & harus & kudu & kudu \\
\hline 50. & dandan & dangdan & dangdan \\
\hline 51. & punya & duwe & duwe \\
\hline 52. & beli & tuku & tuku \\
\hline 53. & dipukul & digebug & digebug \\
\hline 54. & menghina & ngece & ngece \\
\hline 55. & menguap & angob & angob \\
\hline 56. & mencuri & nyolong & nyolong \\
\hline 57. & pakai & nggo & nggo \\
\hline 58. & mau/akan & pengen & pengen \\
\hline 59. & jangan & aja & aja \\
\hline
\end{tabular}




\begin{tabular}{|l|l|l|l|}
\hline 60. & tanya & nakon & nakon \\
\hline 61. & pacaran & demenan & demenan \\
\hline 62. & butuh & butuh & butuh \\
\hline 63. & lupa & klalen & klalen \\
\hline 64. & ingat & inget & inget \\
\hline 65. & minum (meminum) & nginum & nginum \\
\hline 66. & makan (memakan) & mangan & mangan \\
\hline 67. & gigit (menggigit) & cokot & cokot \\
\hline 68. & nafas (bernafas) & ambekan & ambekan \\
\hline 69. & lihat (melihat) & deleng & deleng \\
\hline 70. & tahu (mengetahui) & weru & weru \\
\hline 71. & pikir (berpikir) & mikir & mikir \\
\hline 72. & bau (membau) & mambu & mambu \\
\hline 73. & takut & wédi & wédi \\
\hline 74. & tidur & turu & turu \\
\hline 75. & hidup & urip & urip \\
\hline 76. & mati & mati & mati \\
\hline 77. & potong (memotong) & tugél (nyembeleh) & tugél \\
\hline 78. & terbang & mabur & mabur \\
\hline 79. & jalan (berjalan) & mlaku & mlaku \\
\hline 80. & datang & teka & teka \\
\hline 81. & diri (berdiri) & ngadeg & ngadeg \\
\hline 82. & jatuh & tiba & tiba \\
\hline 83. & tarik (menarik) & tarik & tarik \\
\hline 84. & dorong (mendorong) & dorong & dorong \\
\hline 85. & nyanyi (bernyanyi) & némbang & némbang \\
\hline 86. & main (bermain) & dolan & dolan \\
\hline & & & \\
\hline
\end{tabular}

\section{Adjective}

The study found 69 same words and 14 different words in the form of nouns used in Pabuaran and Ciperna. Those words are displayed in the table below:

Table 3.5 Adjective

\begin{tabular}{|c|c|c|c|}
\hline No. & ARTI & $\begin{array}{c}\text { DIALEK } \\
\text { PABUARAN }\end{array}$ & DIALEK CIPERNA \\
\hline 1. & pelit & ala & medit \\
\hline 2. & kesel & enek & mengkel \\
\hline 3. & jelek & bapu & blesak \\
\hline 4. & sedikit & secuil & setitik \\
\hline 5. & rusak & bodol & rusak \\
\hline 6. & terserah & pajareh & karep \\
\hline 7. & benci & blih seneng & nyengit \\
\hline 8. & kiri & kede & kiwe \\
\hline 9. & tebal & kandél & tebél \\
\hline 10. & pendek & cindék & pendek \\
\hline 11. & sempit & sempit & sésék \\
\hline 12. & tipis & ipis & tipis \\
\hline 13. & buruk & bapu & blésak \\
\hline 14. & lurus & lémpéng & lurus \\
\hline 15. & cantik & ayu & ayu \\
\hline 16. & ramah & ramah & ramah \\
\hline 17. & sombong & sombong & sombong \\
\hline 18. & rajin & rajin & rajin \\
\hline 19. & kasar & akas & akas \\
\hline 20. & lincah & lincah & lincah \\
\hline 21. & lucu & lucu & lucu \\
\hline
\end{tabular}




\begin{tabular}{|c|c|c|c|}
\hline 22. & lambat & sue & sue \\
\hline 23. & gemulai & kemayu & kemayu \\
\hline 24. & malas & males & males \\
\hline 25. & nakal & nakal & nakal \\
\hline 26. & parah & parah & parah \\
\hline 27. & sepi & sepi & sepi \\
\hline 28. & sopan & apik & apik \\
\hline 29. & tenang & meneng & meneng \\
\hline 30. & suka & seneng & seneng \\
\hline 31. & marah & nyewot & nyewot \\
\hline 32. & terang & padang & padang \\
\hline 33. & gelap & peteng & peteng \\
\hline 34. & cepat & gelis & gelis \\
\hline 35. & cekatan & rikat & rikat \\
\hline 36. & manis & manis & manis \\
\hline 37. & diam & anteng & anteng \\
\hline 38. & asam & asem & asem \\
\hline 39. & benar & bener & bener \\
\hline 40. & asin & asin & asin \\
\hline 41. & bawah & sor & sor \\
\hline 42. & atas & duwur & duwur \\
\hline 43. & luar & jaba & jaba \\
\hline 44. & selesai & pragat & pragat \\
\hline 45. & susah & angel & angel \\
\hline 46. & bersih & bersih & bersih \\
\hline 47. & putus & tugel & tugel \\
\hline 48. & bingung & keder & keder \\
\hline 49. & bengkok & bengkok & bengkok \\
\hline 50. & lain & séjen & séjen \\
\hline 51. & satu & siji & siji \\
\hline 52. & dua & dua & dua \\
\hline 53. & tiga & telu & telu \\
\hline 54. & empat & papat & papat \\
\hline 55. & lima & lima & lima \\
\hline 56. & besar & gede & gede \\
\hline 57. & panjang & dawa & dawa \\
\hline 58. & lebar & amba & amba \\
\hline 59. & berat & abot & abot \\
\hline 60. & kecil & cilik & cilik \\
\hline 61. & merah & abang & abang \\
\hline 62. & hijau & ijo & ijo \\
\hline 63. & kuning & kuning & kuning \\
\hline 64. & putih & putih & putih \\
\hline 65. & hitam & iréng & iréng \\
\hline 66. & hangat & angét & angét \\
\hline 67. & dingin & atis & atis \\
\hline 68. & penuh & kebék & kebék \\
\hline 69. & baru & anyar & anyar \\
\hline 70. & lama, tua & sue & sue \\
\hline 71. & baik & baik & baik \\
\hline 72. & busuk & bosok & bosok \\
\hline 73. & kotor & bélok & bélok \\
\hline 74. & bulat & bunder & bunder \\
\hline 75. & tajam & landép & landép \\
\hline 76. & tumpul & kétul & kétul \\
\hline 77. & halus & alus & alus \\
\hline 78. & basah & télés & télés \\
\hline
\end{tabular}




\begin{tabular}{|c|l|l|l|}
\hline 79. & kering & garing & garing \\
\hline 80. & betul & bener & bener \\
\hline 81. & dekat & parék & parék \\
\hline 82. & jauh & adoh & adoh \\
\hline 83. & kanan & tengen & tengen \\
\hline
\end{tabular}

\section{Subdialect}

The description above shows that the study found 108 lexical differences and 243 lexical similarities. By using this numbers, then it is run the dialectometric formula as follow:

$$
\frac{\mathrm{S} \times 100}{\mathrm{n}}=\mathrm{d} \% \quad \frac{108 \times 100}{351}=\mathbf{3 0 , 7 6 \%}
$$

Note:

$\mathrm{S}=$ the amount of different words

$\mathrm{n}=$ the amount of words in the instrument

$\mathrm{d}=$ percentage

With the $30,76 \%$, it is then assumed that Pabuaran and Ciperna's lexical variations are chategorized as subdialect of Javanese dialect in Cirebon. This is taken based on the percentage of dialectometric formula as follow:
a. $65 \% \geq \quad$ : assumed as language differences
b. $45-64 \%$ : assumed as dialect differences
c. $25-44 \%$ : assumed as subdialect differences
d. $10-24 \%$ : assumed as speak differences
e. $9 \% \leq \quad$ : assumed as no differences.

\section{Conclusion}

Based on the results of the study and the description above, it can be conluded that: 1) the study found lexical variations of Javanese dialect in Pabuaran and Ciperna region, Cirebon. From 351 words that consist of 207 basic vocabulary by Swadesh and 144 additional words submitted to informants, there are 108 different words used in both region in the form of pronouns (10), nouns (26), verbs (44), adjectives (14), and adverb (14) word classes, 2) based on dialectometric calculation which showed $30,76 \%$, it can be concluded that those differences are categorized as subdialect of Javanese dialect in Cirebon.

\section{References}

Biantoro, L. D. 2009. Dialect Varieties in Tuban, East Java: A Study of Dialect in Kingking and Karangsari. Journal, Surabaya, Former Student of The Department of English Literature, Faculty of Humanities, Airlangga University, 2.

Chaer, Abdul. 2010. Sosiolinguistik. Jakarta: Rineka Cipta.

Maharani, P. D., \& Chandra, K. D. P. 2018. Variasi Leksikal Bahasa Bali Dialek Kuta Selatan. Mudra Jurnal Seni Budaya, 33(1), 76-84.

Nesti, M. R. 2016. Variasi Leksikal Bahasa Minangkabau di Kabupaten Pesisir Selatan. Jurnal Arbitrer, 3(1), 46-61.

Purwaningsih, A. 2017. Geografi Dialek Bahasa Jawa Pesisiran di Desa Paciran Kabupaten Lamongan. In Proceeding of International Conference on Art, Language, and Culture (pp. 594-605). 
Rina, N., \& Mariati, M. 2018. Hubungan Kekerabatan Bahasa Minangkabau Tapan dengan Bahasa Kerinci Sungai Penuh. Jurnal Gramatika: Jurnal Penelitian Pendidikan Bahasa dan Sastra Indonesia, 4(1), 1-11.

Wardaugh, R. 2002. An Introduction to Sociolinguistics. London: Blackwell. 